\title{
An Adaptive Sensorless Control for Permanent Magnet Synchronous Motor Drive in Eelectric Vehicle
}

\author{
Shaofang $\mathrm{Wu}^{\mathrm{a},{ }^{*}, \text { Jianwu Zhang }}{ }^{\mathrm{b}}$ and Xiwen Wang ${ }^{\mathrm{c}}$, Linyong Chen ${ }^{\mathrm{d}}$ \\ National Engineering Laboratory for Automotive Electronics and Control Technology, Shanghai \\ Jiaotong University, Shanghai 200240, China \\ ashaofangwu@sjtu.edu.cn, bjianwuzhang@sjtu.edu.cn, cxiwenwang@sjtu.edu.cn, \\ dlinyongchen@sjtu.edu.cn
}

Keywords: Adaptive observer, sensorless, permanent magnet, electric vehicle.

\begin{abstract}
This paper presents an adaptive sensorless control for permanent magnet synchronous motor in the electric vehicles drive system. Using stator currents, voltage and speed measurements, a new nonlinear adaptive position observer for the permanent magnet synchronous motor is designed. Convergence of the position observer is guaranteed. With the position information provided by the observer, control scheme is implemented in the field-oriented strategy to adjust the motor torque or rotor speed to propel the vehicle. Simulations are carried out to test the sensorless control and results show that the proposed method performs effectively to regulate the drive system of the electric vehicle.
\end{abstract}

\section{Introduction}

Permanent magnet synchronous motors (PMSMs) are widely used in pure electric or hybrid vehicle drives due to their advantages such as high efficiency, high torque density and wide speed range. The electric vehicle drive control is to regulate the torque or speed of the motor to achieve a fast and precise response according to the driver's commands. In the recent years, the sensorless control is receiving extensive attention. Its philosophy of eliminating the position sensors is of significance in electric vehicle applications. Because the position sensors are vulnerable to harsh environment conditions [1], removal of mechanical sensors increases reliability besides saving volume, wires, cost. In the sensorless control, observers are utilized as 'soft sensors' to provide the position information, with stator currents or voltages as the available measurements.

Many articles are available for the position or speed estimation of PMSMs sensorless control. A nonlinear Luenberger observer for PMSMs is proposed in [2] which estimates both the rotor position and the flux. An online identification for the resistance to improve the control robustness with respect to resistance uncertainties is presented in [3]. A discrete-time variable structure control is considered and the rotor position and speed are obtained through an adaptive extended Kalman filter in [4]. A field-oriented adaptive sensorless control for the regulation of the speed and field of PMSMs drives is proposed in [5]. An adaptive controller based on input-output feedback linearization for speed and torque control of sensorless PMSMs is developed for electric vehicle application in [6]. Reference [7] introduces an adaptive inverse control strategy for PMSMs drive system and proves that the algorithm is effective and satisfies the need of micro-electric vehicle drive control. An adaptive fuzzy active disturbance rejection control design technique on PMSMs for electric vehicle is proposed in [8].

In this paper, a nonlinear adaptive position observer is presented to estimate the rotor angle position. And the control is implemented in simulations to adjust the motor to propel the vehicle.

\section{Modelling of PMSM Drive}

The model for PMSMs in the rotating direct-quadrature $(\mathrm{d}-\mathrm{q})$ frame is presented as follows

$$
\left\{\begin{array}{l}
L_{d} \frac{d i_{d}}{d t}=-R_{s} i_{d}+L_{q} \omega_{e} i_{q}+u_{d} \\
L_{q} \frac{d i_{q}}{d t}=-R_{s} i_{q}-L_{d} \omega_{e} i_{q}-\psi \omega_{e}+u_{q} \\
\frac{J}{n} \frac{d \omega_{e}}{d t}=n\left[\psi i_{q}+\left(L_{d}-L_{q}\right) i_{d} i_{q}\right]-\frac{B}{n_{p}} \omega_{e}-T_{L}
\end{array}\right.
$$


where $i_{d}$ and $i_{q}$ are d- and q-axis currents; $\omega_{e}$ is electrical angular velocity; $u_{d}$ and $u_{q}$ are d- and qaxis voltages. $L_{d}$ and $L_{q}$ are inductances in d-q frame; $R_{s}$ is stator resistance; $B$ is coefficient of friction, and $\psi$ is the permanent magnet flux. $n_{p}$ is number of pole pairs; $J$ is moment of inertia and $T_{L}$ is load torque. For the case of surface-mount PMSMs, $L_{d}=L_{q}$.

\section{Position Observer Design}

The observer is designed in the stationary frame to provide rotor position, required in the fieldoriented control. The representation for SPMSMs in the fixed $\alpha$ - $\beta$ frame can be presented as follows

$$
\begin{aligned}
& L \frac{d i_{\alpha \beta}}{d t}=-R i_{\alpha \beta}-n_{p} \omega J \phi \rho_{\alpha \beta}+v_{\alpha \beta} \\
& J \dot{\omega}=n_{p} \psi i_{\alpha \beta}^{T} J C(\theta)-\tau_{L} \\
& \dot{\theta}=n_{p} \omega
\end{aligned}
$$

Where $i_{\alpha \beta}=\left[i_{\alpha}, i_{\beta}\right]^{T}$ and $v_{\alpha \beta}=\left[v_{\alpha}, v_{\beta}\right]^{T}$ are stator currents and voltages, $\omega$ is angular velocity; $\theta$ is angular position; $L$ is stator inductance. $\operatorname{And} C(\theta):=[\cos \theta, \sin \theta]^{T}, \mathcal{J}:=\left[\begin{array}{cc}0 & -1 \\ 1 & 0\end{array}\right]$.

In the SPMSMs, the stator flux $\lambda$ is related with the currents and voltages via

$\lambda=L i_{\alpha \beta}+\psi \rho_{\alpha \beta}$

Furthermore, the following relation would be helpful in the construction of the observer

$\dot{C}(\theta)=n_{p} \omega J C(\theta)$

It is well known that the rotor angle position can be obtained from the estimated flux since the rotor position is encrypted in the total flux. Further, it can also be found that the rotor position is also encrypted in the components of the flux due to the permanent magnet. Therefore, we can construct an adaptive observer to estimate the rotor position directly based on the components of permanent magnet flux. Hence, the adaptive position observer is presented as follows

$$
\begin{aligned}
& \dot{\xi}_{12}=-2 n_{p} \omega \mathcal{J}\left[\mathcal{T}-L i_{\alpha \beta}\right]+2 n_{p} \omega \mathcal{J} \hat{\eta}_{12}-\frac{\partial \beta}{\partial i_{\alpha \beta}} L^{-1}\left\{v_{\alpha \beta}-R i_{\alpha \beta}+n_{p} \omega \mathcal{J}\left[\mathcal{T}+\hat{\eta}_{12}\right]\right\} \\
& \hat{\eta}_{12}=\xi_{12}+\beta_{12}\left(i_{\alpha \beta}\right)
\end{aligned}
$$

where $\mathcal{T}=\int_{t_{0}}^{t}\left(v_{\alpha \beta}-R i_{\alpha \beta}\right) d t+\lambda_{0}$ with $t_{0}$ the initial time. $\xi_{12} \in \mathcal{R}^{2}$ is the observer state and $\beta_{12}: \mathcal{R}^{2} \rightarrow \mathcal{R}^{2}$ is a mapping defined by $\beta_{12}=\left[-2 L\left(i_{\alpha}+\Gamma \omega i_{\beta}\right), 2 L\left(i_{\beta}+\gamma \omega i_{\alpha}\right)\right]^{T}$ with some positive scalars $\Gamma$ and $\gamma$, and $\hat{\eta}_{12}$ is the observer output.

This observer ensures boundedness of $\xi_{12}$ and $\hat{\eta}_{12}$ and guarantees global asymptotic convergence of $\hat{\eta}_{12}$ towards its actual values, that is $\lim _{t \rightarrow \infty}\left|\hat{\eta}_{12}-\eta_{12}\right|=0$, if and only if $\omega \notin \mathcal{L}^{2}$, and hence the position estimate $\hat{\theta}$ can be obtained as $\hat{\theta}=\arctan \left(\frac{\hat{\eta}_{2}}{\hat{\eta}_{1}}\right)$.

The convergence is to show that $\lim _{t \rightarrow \infty}\left|\hat{\eta}_{12}-\eta_{12}\right|=0$. Here, it can be defined that $\eta_{12}=2 \psi C(\theta)$, which represents the unmeasured parts of the system. Using (4), we can have

$\dot{\eta}_{12}=n_{p} \omega J \eta_{12}$

Denote $z_{12}$ the error variable as $z_{12}=\hat{\eta}_{12}-\eta_{12}$. The time derivative of $z_{12}$ is described by

$$
\dot{z}_{12}=\dot{\xi}_{12}+\frac{\partial \beta_{12} d i_{\alpha \beta}}{\partial i_{\alpha \beta} d t}-\dot{\eta}_{12}
$$

Selecting the functions $\beta_{12}=\left[\beta_{1}, \beta_{2}\right]^{T}=\left[-2 L\left(i_{\alpha}+\Gamma \omega i_{\beta}\right), 2 L\left(i_{\beta}+\gamma \omega i_{\alpha}\right)\right]^{T}$ with $\Gamma, \gamma>0$, and substituting (2), (4), (5), (6) and $\beta_{12}$ into (7), it can be obtained that

$$
\begin{aligned}
\dot{z}_{12} & =-2 n_{p} \omega \mathcal{J}\left[\mathcal{T}-L i_{\alpha \beta}\right]+2 n_{p} \omega \mathcal{J} \hat{\eta}_{12}-\dot{\eta}_{12}-\frac{\partial \beta}{\partial i_{\alpha \beta}} L^{-1}\left\{v_{\alpha \beta}-R i_{\alpha \beta}+n_{p} \omega \mathcal{J}\left[\mathcal{T}+\hat{\eta}_{12}\right]\right\}+\frac{\partial \beta_{12} d i_{\alpha \beta}}{\partial i_{\alpha \beta} d t} \\
& =2 n_{p} \omega \mathcal{J}\left(\hat{\eta}_{12}-\eta_{12}\right)+\left[\begin{array}{cc}
-2 & -2 \Gamma \omega \\
2 \gamma \omega & -2
\end{array}\right] n_{p} \omega \mathcal{J}\left(\hat{\eta}_{12}-\eta_{12}\right)
\end{aligned}
$$

The above equation can be further rewritten as

$$
\dot{z}_{12}=\left[\begin{array}{cc}
-2 n_{p} \Gamma \omega^{2} & 0 \\
0 & -2 n_{p} \gamma \omega^{2}
\end{array}\right] z_{12}
$$

When $\omega \notin \mathcal{L}^{2}$, and $\Gamma, \gamma>0, \lim _{t \rightarrow \infty}\left|z_{12}\right|=\lim _{t \rightarrow \infty}\left|\hat{\eta}_{12}-\eta_{12}\right|=0$. Thus, the claim is established. 


\section{Vehicle and Driver Modelling}

The longitudinal dynamics of electric vehicle is modeled as one-degree of freedom motion, and the vehicle is considered as a point mass $M$ subject to propelling torque and resistant fiction [9]. The dynamical equation is given as follows

$$
\delta M \dot{u}=\frac{T_{w}}{r}-\frac{1}{2} \rho A C_{D} u^{2} \operatorname{sign}(u)-M g[\sin (\alpha)+f \cos (\alpha) \operatorname{sign}(u)]
$$

where $\delta$ is coefficient of rotational inertia to mass, $M$ is vehicle mass, $u$ is vehicle velocity, $T_{w}$ is wheel torque, $r$ is wheel radius, $\rho$ is air density, $A$ is frontal area, $C_{D}$ is aerodynamic drag coefficient, and $g$ is the acceleration of gravity. $f$ is coefficient of rolling resistance, and $\alpha$ is slope along the road.

Parameters of the vehicle are showed in Table 1 and parameters of the motor are given in Table 2. To achieve the objective of following a desired path, i.e., making the vehicle tracking a velocity profile $u^{*}$, the driver operates the pedals to express a wheel torque request $T^{*}[10]$ as

$$
T^{*}=k_{p}\left(u^{*}-u\right)+k_{i} \int_{0}^{t}\left[u^{*}(\tau)-u(\tau)\right] d \tau
$$

Where $k_{p}$ and $k_{i}$ are two scalars. According to the desired speed, the driver model generates respective torque command to adjust the speed of the motor and vehicle.

Table 1. Parameters of the vehicle.

\begin{tabular}{|c|c|c|c|}
\hline Parameters & Values & Parameters & Values \\
\hline Coefficient of rotational mass $\delta$ & 1.01 & Frontal Area $A$ & $1.65 \mathrm{~m}^{2}$ \\
\hline Coefficient of rolling resistance $f$ & 0.012 & Wheel Radius $r$ & $0.27 \mathrm{~m}$ \\
\hline Aerodynamic Drag Coefficient $C_{D}$ & 0.4 & Vehicle Mass $M$ & $1200 \mathrm{~kg}$ \\
\hline
\end{tabular}

Table 2. Parameters of the motor.

\begin{tabular}{|c|c|c|c|}
\hline Parameters & Values & Parameters & Values \\
\hline Stator inductance $L$ & $0.04 \Omega$ & Number of pole pairs & 4 \\
\hline Flux linkage $\psi$ & $0.05 \mathrm{mH}$ & Friction coefficient $B$ & 0.0005396 \\
\hline Stator resistance $R_{S}$ & $0.15 \mathrm{~Wb}$ & & \\
\hline
\end{tabular}

\section{Simulations and Results}

Simulations are carried out to test the sensorless control scheme. The responses of the proposed control in the torque control mode are shown in Figure 1(a) and the responses in the speed mode are presented Figure 1(b). As shown, the electromagnetic torque follows closely to the torque output using a position sensor, and the speed tracks its reference signal closely. The results reveal that the proposed sensorless control scheme is effective to regulate the electromagnetic torque production and achieve precise speed tracking. And the proposed control is possible to be used in the PMSM drive of electric vehicles.

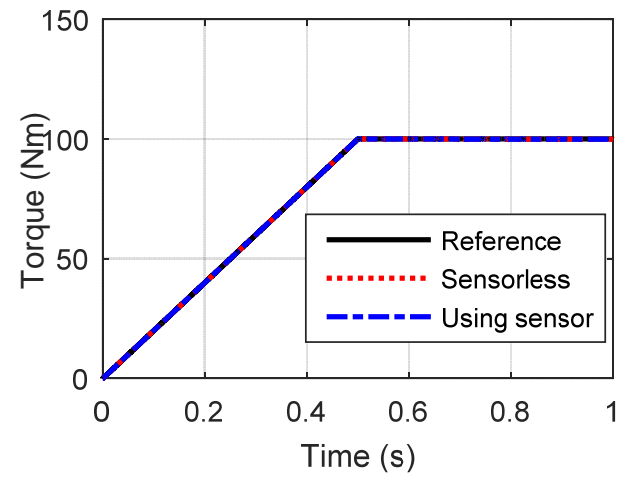

(a)

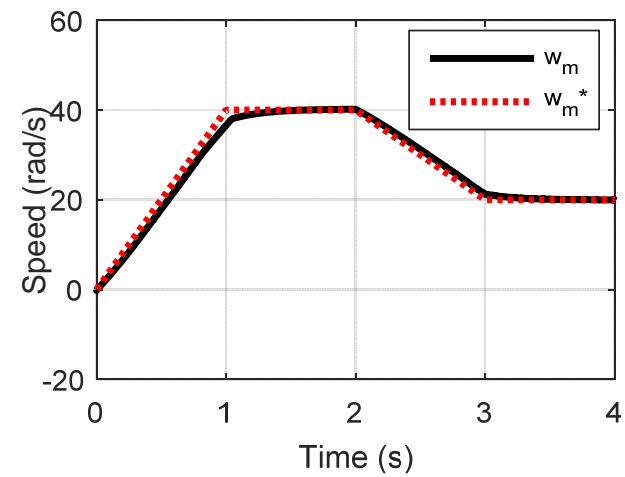

(b)

Figure 1. Comparisons of the torque and speed: (a) torque responses; (b) speed responses. 


\section{Conclusion}

An adaptive sensorless control for PMSM drive in electric vehicle has been presented. The position observer has been designed to provide the rotor angle. The observer is designed using the stator currents, voltages and rotor speed. Convergence of the observation is guaranteed. Simulations have demonstrated that the position-sensorless control is effective to regulate the torque and speed tracking.

\section{References}

[1]. Lin F., Hung Y., Chen J., and Yeh C. (2014) Sensorless IPMSM drive system using saliency back-EMF-based intelligent torque observer with MTPA control, IEEE Trans. on Ind. Inf., 10, 1226-1241.

[2]. Henwood N. , Malaizé J. , and Praly L. (2012) A Robust Nonlinear Luenberger Observer for theSensorless Control of SM-PMSM:Rotor Position and Magnets Flux Estimation, 1625-1630.

[3]. Nahid-Mobarakeh B., Meibody-Tabar F., and Sargos F. (2004) Mechanical snesorless control of pmsm with online estimation of stator resistance, IEEE Trans. on Ind. App., 40, 457-471.

[4]. Ciabattoni L., Corradini M., Grisostomi M., Ippoliti G., Longhi S. and Orlando G. (2011) Adaptive Extended Kalman Filter forRobust Sensorless Control of PMSM Drives, IEEE Conference on Decision and Control and European Control Conference, 934-939.

[5]. Medagam P., Yucelen T., Pourboghrat F. (2009) Adaptive SDRE Based Nonlinear Sensorless Speed Controlfor PMSM Drives, American Control Conference, 3866-3871.

[6]. Oghafy V., Nikkhajoei H. and Zamani J. (2009) An Adaptive Controller for Sensorless PM Synchronous Motor Drive for Electric Vehicles, 2009 IEEE Vehicle Power and Propulsion Conference, 265-269.

[7]. Li R., Zhao J., Hu B. and Xu G. (2009) Adaptive Inverse Control of Permanent Magnet Synchronous Motor Drive in a Micro-Electric Vehicle, International Conference on Machine Learning and Cybernetics, 1909-1914.

[8]. Wen J., Su G. and Cao B. (2009) Fuzzy Adaptive Controller Design of Permanent Magnet Synchronous Motor forElectric Vechicle, 3th International Symposium on Intelligent Information Technology Application, 500-503.

[9]. Soares A., Quintiliano B., and Dias R. (2013) Vehicle Dynamics: A New Way of Understanding and Optimizing Vehicle Performance, SAE Technical Paper, 2013-36-0335.

[10]. S. Zhang and W. Deng, Driver Modeling for Simulation of Transportation Systems, 2012 IEEE Vehicular Technology Conference, (2012)1-7. 